\title{
Nonintrusive measurement of time-resolved emittances of 1-GeV operational hydrogen ion beam using a laser comb
}

\author{
Y. Liu๑, C. Long, and A. Aleksandrov® \\ Spallation Neutron Source, Oak Ridge National Laboratory, Oak Ridge, Tennessee 37831, USA
}

(Received 24 August 2020; accepted 9 October 2020; published 28 October 2020)

\begin{abstract}
We proposed and demonstrated a novel technique to measure time-resolved transverse emittances of the hydrogen ion $\left(\mathrm{H}^{-}\right)$beam in a $1-\mathrm{GeV}$ high-power accelerator. The measurement is performed in a nonintrusive manner by using a laser comb-laser pulses with controllable multilayer temporal structure. The technique has been applied to the transverse emittance measurement of a $1-\mathrm{GeV} \mathrm{H}^{-}$beam in the Spallation Neutron Source high energy beam transport line. More than 20 time-resolved emittances have been simultaneously determined within a macropulse, a single minipulse, or a single bunch of the 1.4-MW neutron production $\mathrm{H}^{-}$beam from a single measurement.
\end{abstract}

DOI: 10.1103/PhysRevAccelBeams.23.102806

\section{INTRODUCTION}

Measurement of transverse phase space distributions is important for improvement of beam matching and control of beam loss in the design and operation of particle accelerators. Conventional transverse phase space measurements use slit-and-collector or pepper-pot approaches [1], both of which intercept the particle beam and cannot be applied to high-power particle beams. Meanwhile, a laserbased nonintrusive emittance and profile measurement system, referred to as laser wire, has been developed in a number of hydrogen ion $\left(\mathrm{H}^{-}\right)$[2-8] or electron accelerators $[9,10]$. The measurement is based on the photodetachment (for ion beam) or the Compton scattering (for electron beam) process. Both processes cause negligible loss to the particle beam and therefore can be applied to the operational beam.

At the Spallation Neutron Source (SNS), the original approach of using the Twiss parameters determined from the emittance measurement to match the $\mathrm{H}^{-}$beam at the injection to the linac or between the linac sections did not prove to be useful. All attempts to set up the SNS accelerator in accordance with the model derived parameters, e.g., matching to the design lattice, ended up with high overall beam loss. Matching the beam core, evidently, does not help to reduce the beam loss. The current focus of beam diagnostics development at SNS is to help in resolving this discrepancy between the computer modeling and the actual

\footnotetext{
*liuy2@ornl.gov

Published by the American Physical Society under the terms of the Creative Commons Attribution 4.0 International license. Further distribution of this work must maintain attribution to the author(s) and the published article's title, journal citation, and DOI.
}

machine operation. The two major directions are improving the dynamic range of the measurements to resolve the part of the beam that is related to the beam loss and increasing the temporal resolution on all scales of the SNS beam structure. The latter is the main subject of this paper.

As illustrated in Fig. 1, the beam in the SNS linac has a complicated time structure spanning a range from picoseconds to a millisecond: a continuous stream of bunches of picosecond duration and $2.48 \mathrm{~ns}$ period are formed by the radio frequency quadrupole; this stream is modulated by the fast chopper to form minipulses of $\sim 1 \mu$ s period and a variable duration in the $0.3-0.8 \mu$ s range; sets of minipulses of variable lengths from 1 to $1000 \mu$ s further form the macropulse, which can repeat at up to $60 \mathrm{~Hz}$ repetition rate. There is no reason to assume that the beam emittance is the same on all time scales. For example, the chopper rise time of $\sim 20$ ns covers several bunches and, as a result, the bunches during the transient of the minipulse could have the charge and diverging angle different from the bunches in the main part of the minipulse. In fact, time-resolved emittance measurement at the SNS front end demonstrated the emittance variation along the minipulse rise time $[11,12]$. The emittance can also vary along the 1-ms macropulse due to the nonuniform beam current from the ion source or the nonuniform pulse profile from the linac rf system. It was unclear whether these effects are important because the existing emittance measurement in the SNS front end cannot measure pulses longer than $50 \mu$ s due to the power limitation of the interceptive instrumentation. It is well known for electron linacs [13] and has been demonstrated recently for hadron beams [14] that the transverse emittance can depend on the temporal position within the bunch (slice emittance) or the energy slice within full 6-d phase space. Therefore, time-resolved measurements with sufficient temporal resolution are valuable for precise interpretation of the measured 


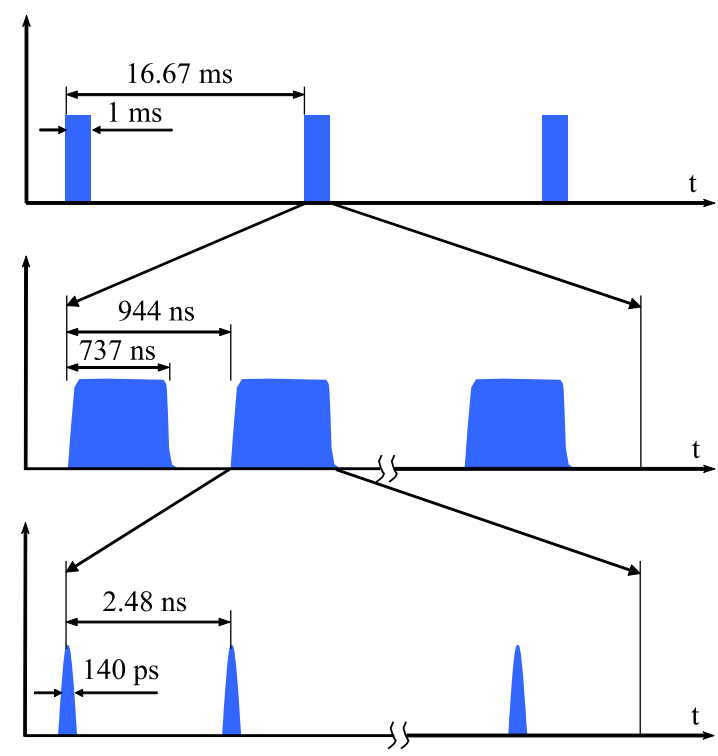

FIG. 1. Typical time structure of the neutron production $\mathrm{H}^{-}$beam macropulses (top), minipulses (middle), and micropulses (bottom) in the high energy beam transport (HEBT) of the SNS accelerator.

transverse emittance, meaningful comparison between measurement and simulation, or comparison between measurements at different locations.

Previously, we have demonstrated the measurement of profile slices within a macropulse of the $\mathrm{H}^{-}$beam by using $10-\mathrm{ns} / 30-\mathrm{Hz}$ laser pulses and appropriately adjusting the timing between laser and ion pulses [6]. In principle, the same technique would also allow to resolve the emittance variations during the minipulse transient and/or along the macropulse. However, the emittance scan is a timeconsuming process and a high-resolution scan typically takes about 30 minutes. Therefore taking a large number of temporal slices would be impractical.

In this paper, we propose a novel technique to simultaneously measure time-resolved emittances of the 1.4-MW operational $\mathrm{H}^{-}$beam. The measurement is based on the use of a laser comb-laser pulses with a designed multilayer temporal structure. We experimentally demonstrate for the first time that more than 20 emittance slices within a macropulse, a single minipulse, or a single bunch of the $\mathrm{H}^{-}$beam can be obtained from one measurement. In the following, we describe the laser comb generation and control, experimental design and signal processing of the time-resolved emittance scan, and measurement results. The method of the nonintrusive measurement of the $\mathrm{H}^{-}$beam transverse phase space using a laser comb is briefly explained in the next section. Section III details the laser system design, implementation and laser comb output. Measurement results on multiple emittance slices within a macropulse, a single minipulse, or a single bunch of the operational $\mathrm{H}^{-}$beam are presented in Sec. IV. Discussions on the longitudinal beam parameter measurement based on the laser comb and the impact of the time-resolved measurement on the accelerator operation are given in the last section.

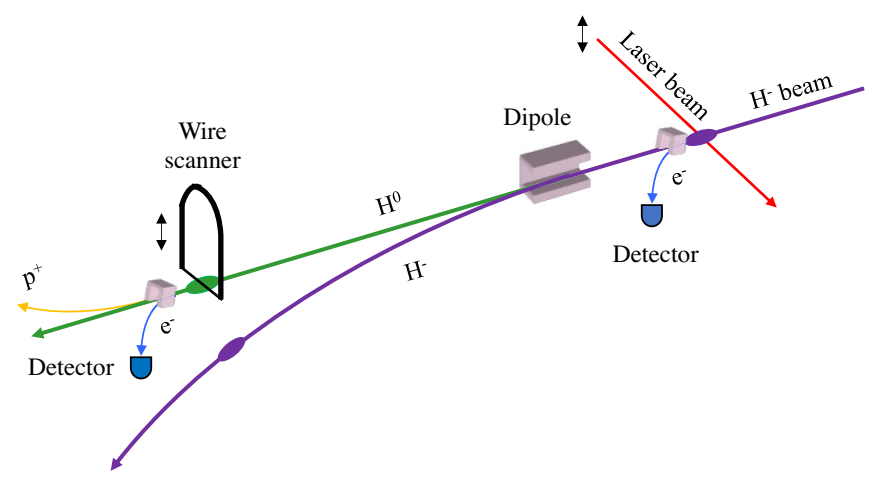

FIG. 2. Schematic of laser-based $\mathrm{H}^{-}$beam transverse emittance measurement.

\section{MEASUREMENT PRINCIPLE}

Figure 2 shows a schematic of the laser wire-based emittance measurement approach. It is in principle a slitdetector emittance scanner except that the conventional slit is replaced by a focused laser beam (laser wire). When the $\mathrm{H}^{-}$beam interacts with a laser light below a certain wavelength $(\sim 1.65 \mu \mathrm{m})$, a small portion of the ions illuminated by the laser are neutralized and the resulting $\mathrm{H}^{0}$ beam is separated from the beam path in a dipole. The distribution of the $\mathrm{H}^{0}$ beam at each laser "slit" or laser wire position is measured by a metal wire (wire scanner) located downstream. The electrons detached from the metal wire are detected by a high-sensitivity detector, in our case a combination of scintillator and photomultiplier tube (PMT). The measured electron density as a function of metal wire and laser wire positions gives the transverse phase space information of the $\mathrm{H}^{0}$ beam. As the $\mathrm{H}^{0}$ beam preserves the same emittance of the original $\mathrm{H}^{-}$beam, the measurement leads to the determination of the $\mathrm{H}^{-}$beam emittance. The laser-based measurement is nonintrusive as the photodetachment typically results in a very small portion $\left(\sim 10^{-5}\right)$ of the loss from the operational $\mathrm{H}^{-}$beam.

An additional advantage of laser-based beam diagnostics is its capability of time-resolved measurement since the laser pulse width can be narrowed to picoseconds, which brings an excellent temporal resolution. The goal of this paper is to demonstrate how multiple, time-resolved emittances can be simultaneously measured using laser pulses with a multilayer temporal structure, referred to as laser comb hereafter. The temporal structure of the laser comb is illustrated in Fig. 3 with the SNS $\mathrm{H}^{-}$beam waveforms as a reference. A typical laser comb used in the experiment contains 20-30 pulse packets (comb teeth) with a controllable comb span and repetition rate of comb teeth. The laser comb is synchronized with the $\mathrm{H}^{-}$beam macropulse and has a repetition rate of $30 \mathrm{~Hz}$ in this work. Each comb tooth contains micropulses which are phase locked to the $\mathrm{H}^{-}$ beam bunches. Such a micropulse structure of the laser comb minimizes the required laser average power, and enhances its capability of phase scan within the $\mathrm{H}^{-}$beam 


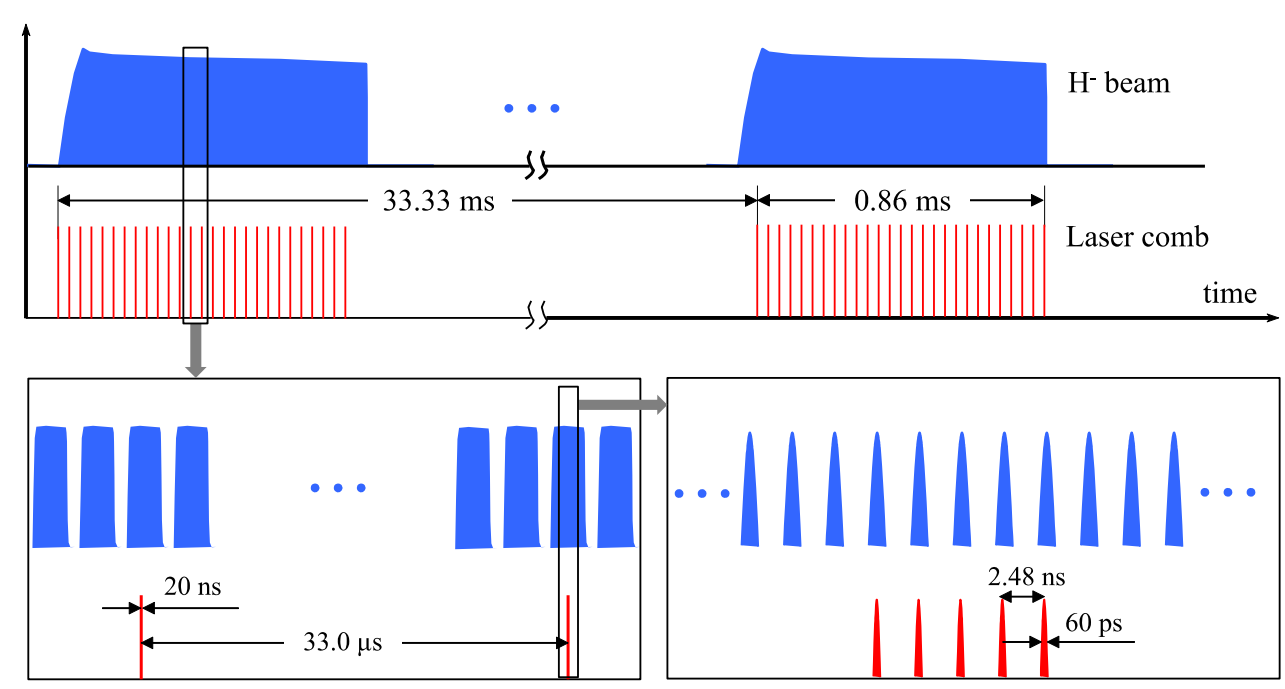

FIG. 3. Temporal structure of the laser comb used for $\mathrm{H}^{-}$beam diagnostics. $\mathrm{H}^{-}$beam waveforms (Fig. 1 is referred to for labels) are plotted as a reference. Top: $\mathrm{H}^{-}$beam macropulse at $60 \mathrm{~Hz}$ and laser comb macropulses at $30 \mathrm{~Hz}$ in this work. Bottom left: waveforms of $\mathrm{H}^{-}$beam minipulses and laser comb teeth. Bottom right: waveforms of $\mathrm{H}^{-}$beam bunches and micropulses inside a laser comb tooth.

bunch. During the emittance measurement, individual laser comb teeth interact with different time sheets of the $\mathrm{H}^{-}$ beam and create time-resolved $\mathrm{H}^{0}$ beam slits. After separation from the $\mathrm{H}^{-}$beam, the $\mathrm{H}^{0}$ beam slits interact with a wire scanner downstream and the stripped electrons are detected. The detection is gated to the laser comb teeth so that the detector outputs from individual temporal slices of the $\mathrm{H}^{-}$beam are properly processed to reconstruct the emittance of the corresponding slice.

An important feature of the SNS $\mathrm{H}^{-}$beam waveforms is that the $402.5 \mathrm{MHz}$ bunch structure is not synchronized in any way with the chopper timing and hence is independent from the minipulse or macropulse structure. Therefore, we will use word "phase" when referring to the bunch time structure and "timing" when referring to the minipulse and macropulse envelopes. Specifically, the laser micropulses are in phase with the ion beam bunches when their repetition frequencies are identical and relative positions of the peaks are constant.

\section{LASER COMB GENERATION AND CONTROL}

The laser comb is generated from a customized hybrid fiber-solid state laser system at a master oscillator power amplifier configuration $[15,16]$ which consists of a seed laser, a fiber-based preamplifier, pulse picker, and solidstate power amplifiers. The comb structure is formed by two stages of pulse picking while the micropulses within individual comb teeth are generated from a fiber-based mode-locked laser. Figure 4 shows the optical setup and key electronic components of the developed laser system. The master oscillator is an actively mode-locked laser. The center laser wavelength is stabilized at $1064.5 \mathrm{~nm}$ and the pulse width of the laser is tunable within 55-85 ps. The pulse repetition frequency is phase locked to the
402.5 MHz reference clock with the phase delay controlled by a signal generator (SG) through a phase shifter within an accuracy of 0.3 ps. The seed laser output is coupled to a high-bandwidth electro-optic modulator (EOM) for the pulse pattern formation. Using a bias controller (YY Labs), we can modulate the seeder output using the rf signal from SG2 at an extinction ratio of $40 \mathrm{~dB}$. The EOM output is preamplified by a ytterbium-doped fiber amplifier (YDFA) which brings the (average) laser power from $\sim 0.5$ to $\sim 50 \mathrm{~mW}$. No stimulated Brillouin scattering oscillations were observed in the YDFA.

The laser output from the YDFA is collimated into a freespace acousto-optic modulator (AOM). The AOM is controlled by the rf signal from SG3 to select a sector of the laser pulses as a laser comb tooth. The AOM control signal is further gated to match the macropulses of the $\mathrm{SNS} \mathrm{H}^{-}$ beam. Figure 5 shows the two rf waveforms applied to the $\mathrm{AOM}$ and EOM, respectively. The comb span and comb teeth spacing are determined by SG3 while the individual comb tooth width is defined by SG2. As detailed in Fig. 5(b), the SG2 output further contains two pulse sequences $(A$ and $B$ ) alternating with each other at the same repetition rate $211.932 \mathrm{kHz}$ that is the fifth subharmonic of the $\mathrm{H}^{-}$beam minipulse frequency. Pulse sequence $A$ consists of narrow (20 ns) pulses that define the width of the laser comb teeth while the pulse sequence $B$ consists of wide (900 ns) pulses. Pulse sequence $B$ is designed to increase the duty factor of the EOM output to $>18 \%$, which is important to avoid parasitic lasing or destructive self-pulsations in the YDFA [15]. It is clear from Fig. 5(b) that only pulses in the sequence $A$ are selected by the AOM to form the laser comb. The rf pulse width of the AOM is set at $1 \mu \mathrm{s}$ which is much wider than both the comb tooth width (20 ns) and the response time ( $200 \mathrm{~ns})$ of the AOM driver. The large pulse width is 


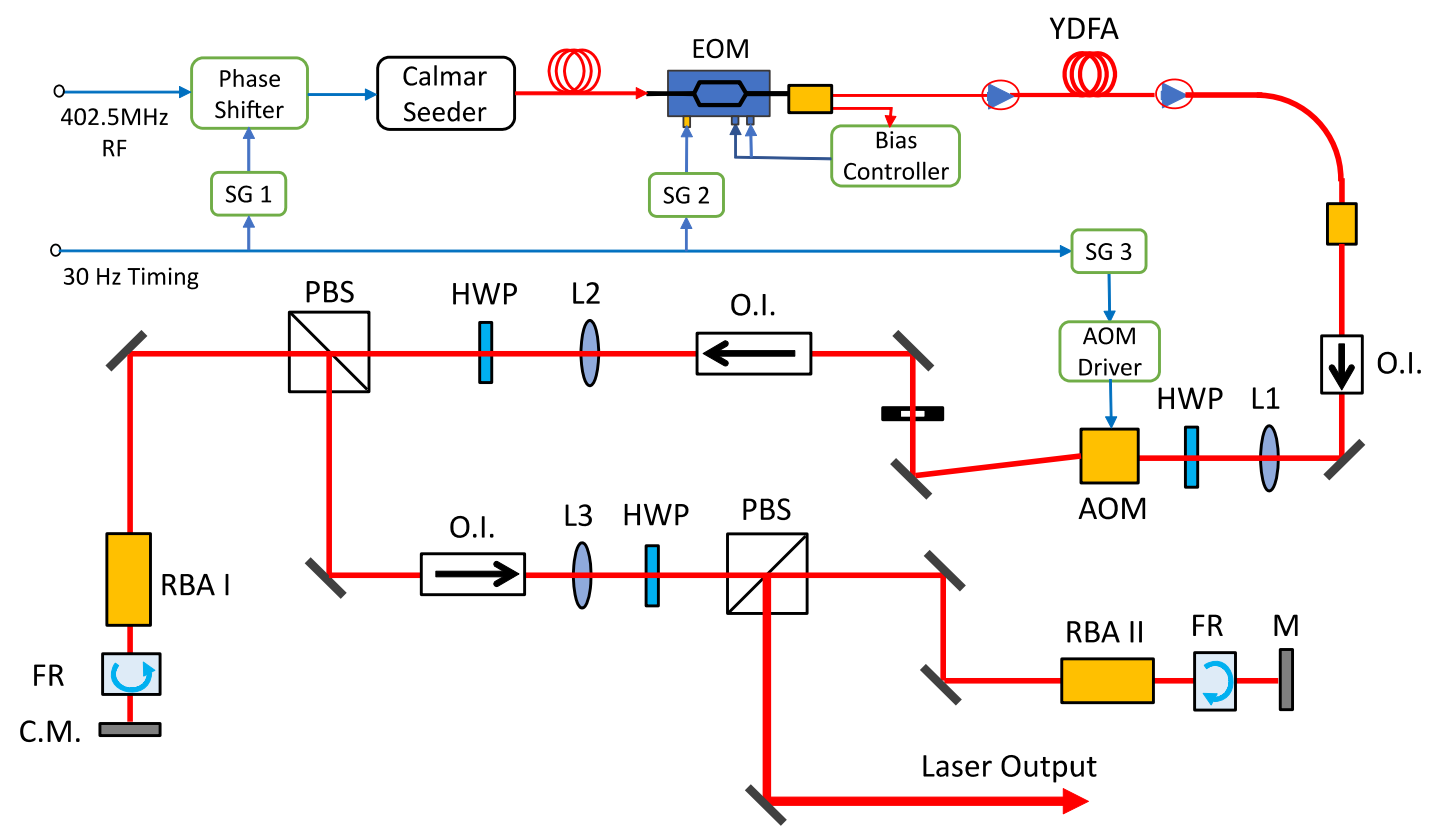

FIG. 4. Schematic of laser system. AOM: acousto-optic modulator; O.I.: optical isolator; L: lens; HWP: half wave plate; M: mirror; C.M.: curved mirror; PBS: polarization beam splitter; FR: Faraday rotator; RBA: diode-pumped Nd:YAG amplifier module; SG1: signal generator for phase shifter control; SG2: signal generator for EOM control; SG3: signal generator for AOM control.

chosen to remove the transient effects of the AOM driver and hence to optimize the transmission of the comb pulses through the AOM.

The formed laser combs are subsequently amplified by two stages of double-pass diode-pumped Nd:YAG amplifiers (Northrop Grumman RBAT24). The gain medium is a $2 \mathrm{~mm}$-diameter, $4 \mathrm{~cm}$-long Nd:YAG rod. The light after the first pass is propagated through a Faraday rotator and then reflected by a curved mirror (radius of curvature of $3 \mathrm{~m}$ ). The reflected light beam, after being rotated $90^{\circ}$ in polarization and amplified through the rod, is reflected by the polarization beam splitter (PBS) and redirected to the next stage. A similar scheme is used in the second amplification stage except that a flat end reflector is used instead of the curved mirror to avoid overfocusing of the laser beam at a higher power. Both amplifiers are pumped by diode bars and the driving current is supplied from a programmable driver that provides the required pulse width and repetition rate according to the designed laser comb setting.

An example of the laser comb waveform is shown in Fig. 6. Figure 6(a) shows the laser combs in a macropulse structure with the macropulse repetition rate of $30 \mathrm{~Hz}$. Figure 6(b) illustrates the structure of individual laser comb. The detailed micropulse structure within a comb tooth is revealed in Fig. 6(c). The full width at half maximum of the micropulse is estimated to be $60 \mathrm{ps}$ (corresponding to $\sim 25 \mathrm{ps}$ rms pulse width). We notice a monotonic decrease of the pulse amplitude in Fig. 6(c). This is due to the gain depletion in the Nd:YAG rod as the front laser pulses extract more gain accumulated in the medium than the later ones. While a flat waveform can be obtained using a pulse shaping modulation scheme
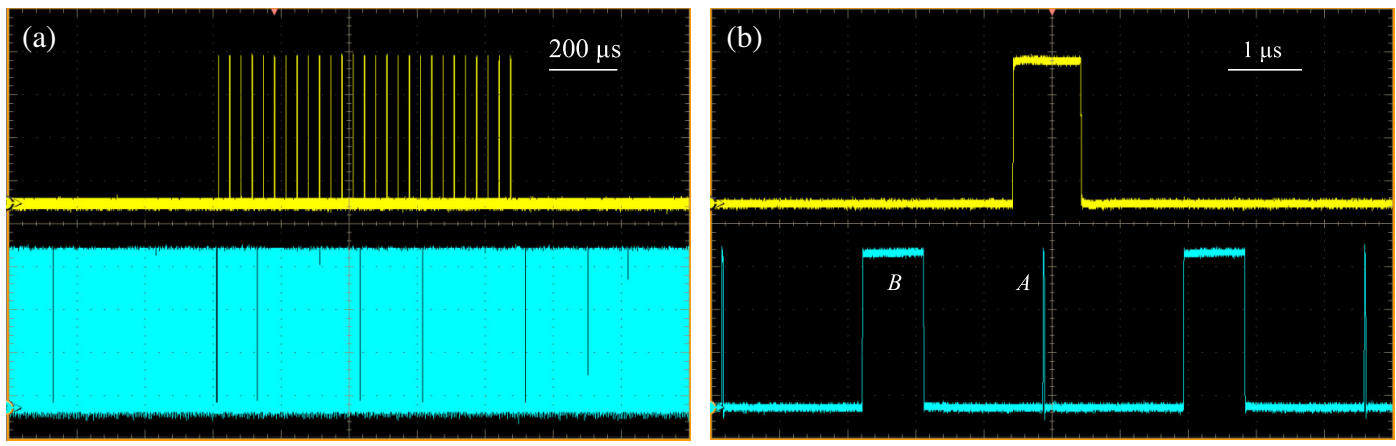

FIG. 5. Rf waveforms corresponding to (a) laser comb macropulse and (b) individual comb tooth. In each figure, the top waveform is the rf signal applied to AOM while the bottom waveform is the rf signal applied to EOM. $A$ and $B$ in (b) represent $20 \mathrm{~ns}$ and $900 \mathrm{~ns}$ pulse sequences, respectively. 

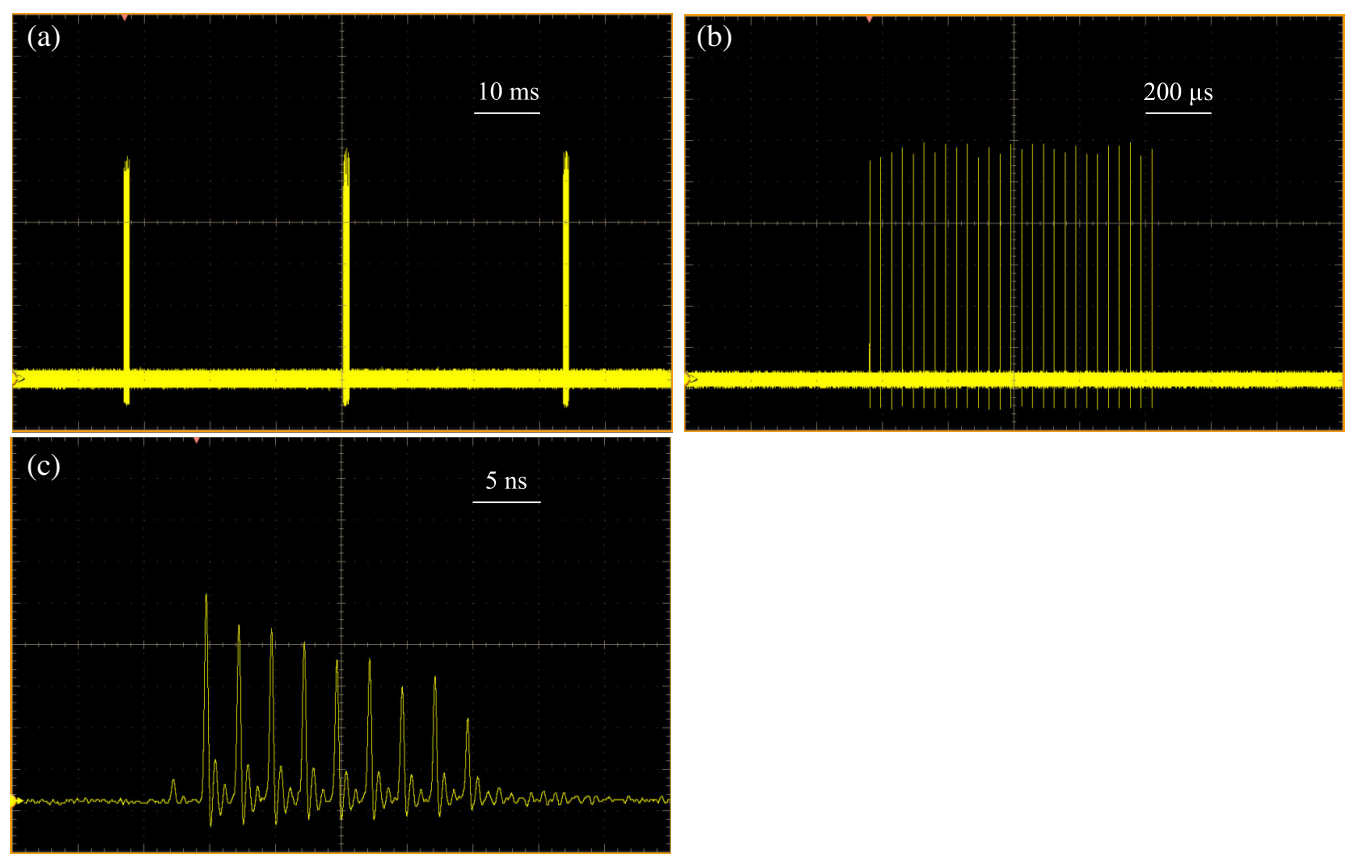

FIG. 6. Laser comb waveforms. (a) 30-Hz macropulses, (b) individual laser comb with a comb span of 35 minipulses ( 33 us) consisting of $27 \mathrm{comb}$ teeth, (c) micropulses within one laser comb tooth. Overshoot in the waveforms is due to the limited bandwidth of the photodetector.

discussed in the previous work [16], for the laser-based $\mathrm{H}^{-}$ beam diagnostics, the nonflat micropulse waveform will not affect the measurement results. This is because the electron detector cannot resolve electrons stripped by individual laser micropulses but rather integrates all electrons over an entire 20-ns time period of the comb tooth. The average laser output power is measured to be $3.5 \mathrm{~W}$, corresponding to a laser peak power of about $6.8 \mathrm{MW}$. For narrower laser micropulses or comb teeth, higher peak power will be achieved from the laser amplifier at a given amplifier setting. Since the photodetachment fraction of an $\mathrm{H}^{-}$beam bunch is typically a few percent which is far below the saturation level, the variation of the laser peak power and hence the variation of the laser pulse width within a range of 50\%$200 \%$ will not affect the emittance measurement performance due to our detection scheme. We measured the laser power fluctuation to be $1.4 \%$ over a five-hour time period. The individual comb teeth amplitude fluctuations are within $2 \%-4 \%$. The spatial profile of the laser beam shows an excellent Gaussian distribution with the beam quality factor $\mathrm{M}^{2}$ measured to be around 1.5.

\section{MEASUREMENT RESULTS}

\section{A. Measurement setup}

Emittance measurements using the developed laser comb have been conducted at the HEBT section of the SNS accelerator. Figure 7 shows the layout of the measurement location. The laser is placed outside the accelerator tunnel and the laser beam is delivered to the measurement station through a 45-meter long free-space laser transport line. At the measurement station, the laser beam is first switched between horizontal or vertical scanning boxes by a remotely controllable flipper mirror. Each scanning box consists of a receiving mirror and a focusing lens mounted on a linear translation stage. A weakly focused laser beam is sent to the laser-ion interaction chamber. At the interaction point, the estimated laser beam diameter is about $80 \mu \mathrm{m}$ and the laser peak power is about $3.5 \mathrm{MW}$. A narrow slice of the $\mathrm{H}^{-}$beam is partially neutralized as a result of the photodetachment due to the focused laser beam, which creates an $\mathrm{H}^{0}$ beam slit. Electrons stripped by the laser beam are steered by a magnet into a Faraday cup placed next to the laser-ion interaction point. The output signals from the Faraday cup are used to produce $\mathrm{H}^{-}$beam profiles at the interaction point. Parameters of the $\mathrm{H}^{-}$beam and a typical laser comb setting used in the measurements are listed in Table I. Using these parameter values, we calculated that $2.4 \%$ of the $\mathrm{H}^{-}$beam illuminated by the laser beam is neutralized [6], which corresponds to a total $\mathrm{H}^{-}$beam fraction of about $1.1 \times 10^{-5}$. Therefore, the influence of the photodetachment on the neutron production is negligible.

After the laser-ion interaction chamber, the particle beam (a mix of $\mathrm{H}^{-}$and $\mathrm{H}^{0}$ ) passes through a dipole magnet. The majority of the beam is undetached $\mathrm{H}^{-}$ions that propagate along the original orbit. The $\mathrm{H}^{0}$ slice, on the other hand, receives no influence from the magnetic field and follows a straight line into the linac dump where a wire scanner is located at 11.6 meters from the laser-ion interaction point. The titanium wires in the wire scanner detach the electrons 


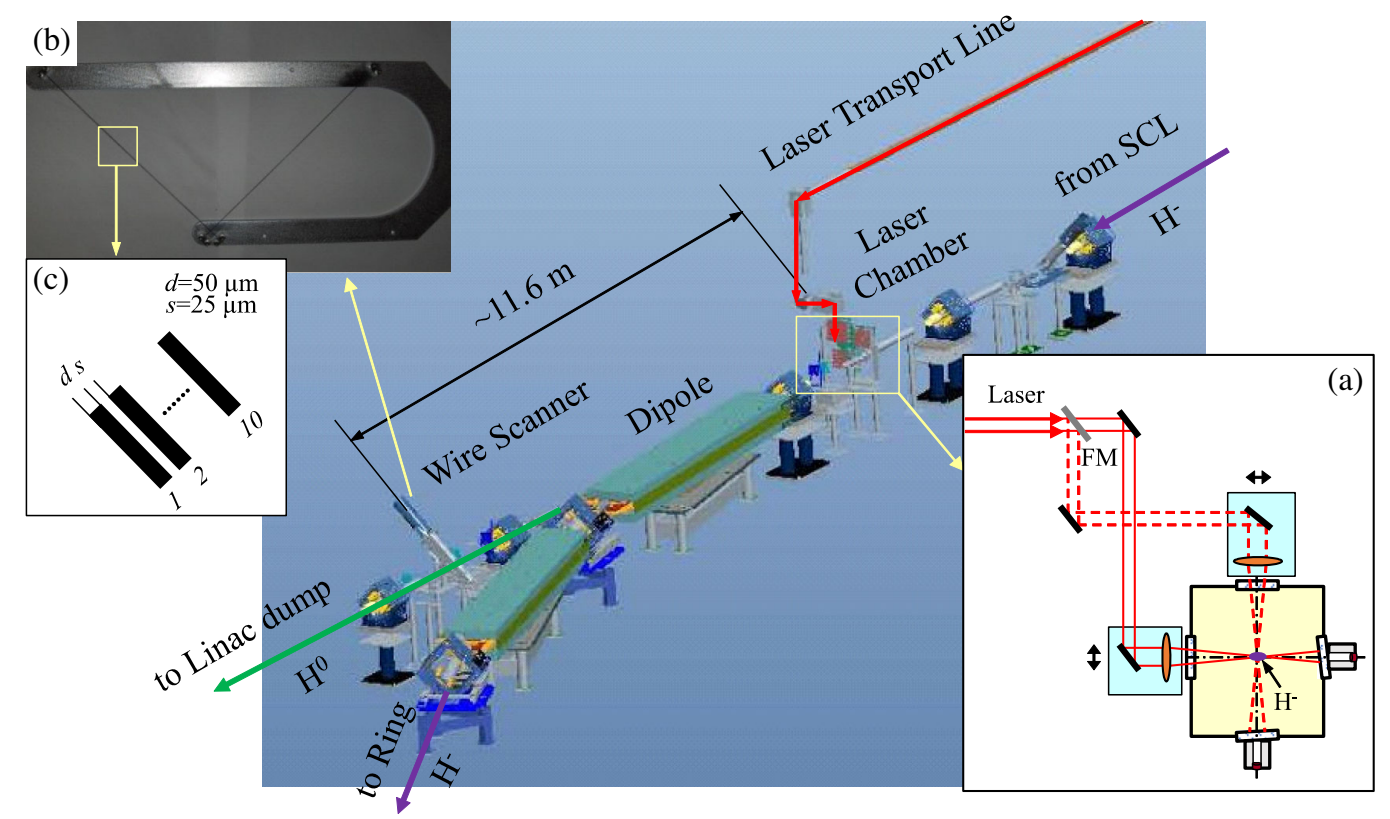

FIG. 7. Location of laser comb-based $\mathrm{H}^{-}$beam emittance measurement station in the SNS HEBT. Inset A shows the laser wire setup while insets $\mathrm{B}$ and $\mathrm{C}$ show the picture and dimension drawings of the ten-wire titanium wire scanner. FM: flipper mirror.

from the $\mathrm{H}^{0}$ beam and the detached electrons are steered to a scintillator by a small magnet. Finally, photons emitted from the scintillator are detected by the PMT.

\section{B. Signal processing}

Both the Faraday cup and PMT output signals are digitized using a National Instruments (NI) PCI-5124 card

TABLE I. Parameters of the $\mathrm{H}^{-}$beam at the emittance measurement station and typical laser comb setting used in the experiments. Some laser comb parameters are adjusted for certain measurements.

\begin{tabular}{lc}
\hline \hline Parameter & Value \\
\hline & $\mathrm{H}^{-}$beam \\
Energy & \\
Average power & $1 \mathrm{GeV}$ \\
Bunch frequency & $1.4 \mathrm{MW}$ \\
rms bunch width & $602.5 \mathrm{MHz}$ \\
Minipulse frequency & $60 \mathrm{ps}$ \\
Minipulse length & $1059.66 \mathrm{kHz}$ \\
Macropulse repetition rate & $737 \mathrm{~ns}$ \\
Macropulse length & $60 \mathrm{~Hz}$ \\
& $1 \mathrm{~ms}$ \\
Wavelength & \\
Peak power & $1064 \mathrm{~nm}$ \\
Micropulse frequency & $3.5 \mathrm{MW}$ \\
rms micropulse width & $402.5 \mathrm{MHz}$ \\
Comb teeth frequency & $\sim 25 \mathrm{ps}$ \\
Comb teeth width & $30.276 \mathrm{kHz}$ \\
Comb repetition rate & $20 \mathrm{~ns}$ \\
Comb span & $30 \mathrm{~Hz}$ \\
\hline \hline
\end{tabular}

at a sampling rate of 200 mega samples/sec. The signal processing is performed using custom software developed by our team in NI LabVIEW (32 bit edition, 2014). When processing signals for emittance measurement, the acquired data are first chopped into groups, each corresponding to a comb tooth. In each group, data is collected for a time equivalent to five minipulses, with data from the laser- $\mathrm{H}^{-}$ beam interaction only in the first minipulse. The last four waveforms are averaged, and the result is subtracted from the waveform of the first minipulse, which effectively reduces the induced spikes in the resulting waveform, leaving only the signal resulting from the laser comb. An example of the Faraday cup and PMT output signals from the laser comb is shown in Fig. 8. Here, each pulse in the figure represents only the region of interest containing the detected signal produced from a given laser comb tooth, the intervening signal between two comb teeth (up to 35 minipulses or $33 \mu \mathrm{s}$ ) is discarded to save storage space. As stated before, due to the bandwidth limitation of the detectors, all electrons generated from one laser comb tooth are detected as one pulse. The PMT output is calculated by integrating the pulse. For emittance measurements, the PMT output at each position of the laser beam and wire scanner is averaged over 15 macropulses to reduce the effect of the laser beam jitter. The phase space distribution is reconstructed by plotting the measured PMT output as a function of the laser and wire scanner positions. Figure 9 shows an example of the phase space distributions in horizontal (a) and vertical (b) planes measured from one laser comb tooth. During the measurement, both laser beam and wire scanner are moved at a step size of $0.5 \mathrm{~mm}$. The corresponding angle resolution is $43 \mu \mathrm{rad}$. 


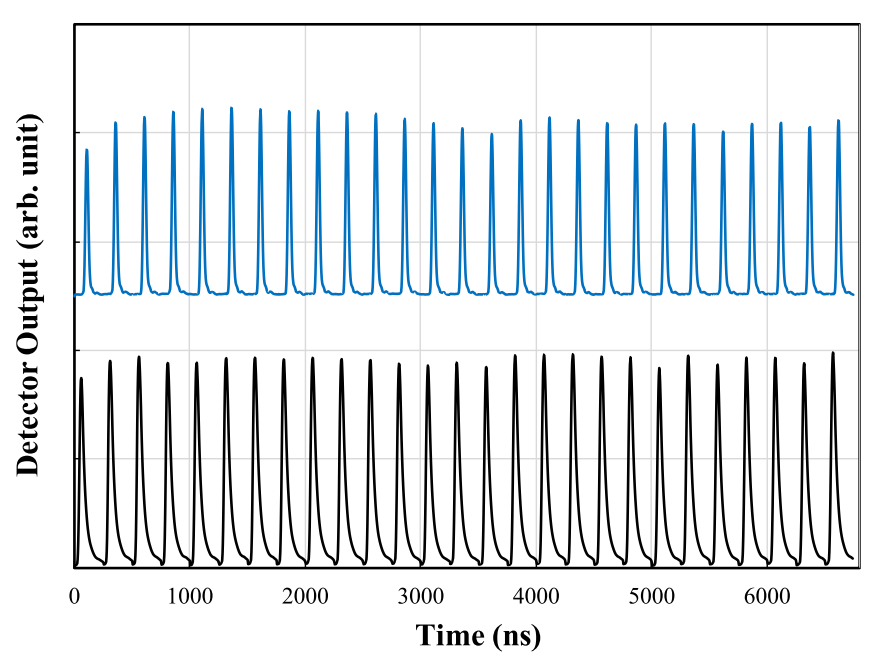

FIG. 8. Output waveforms of the Faraday cup (top) and PMT (bottom) from a laser comb consisting of $27 \mathrm{comb}$ teeth. For each laser comb tooth, only $250 \mathrm{~ns}$ is displayed.

A revised data acquisition driver scheme has been developed to accommodate laser combs with a large span. The modification and additional code optimizations were able to reduce the overhead and allow laser comb data processing to proceed at up to $60 \mathrm{~Hz}$ (repetition rate of the SNS $\mathrm{H}^{-}$beam macropulses) of laser triggering.

\section{Emittance scan over macropulse of the $\mathrm{H}^{-}$beam}

Our first application of the laser comb is the simultaneous measurement of multiple emittance slices across an $\mathrm{H}^{-}$beam macropulse. The measurement can only be performed with the laser method because the other emittance measurements at the SNS intercept beam and

(a)

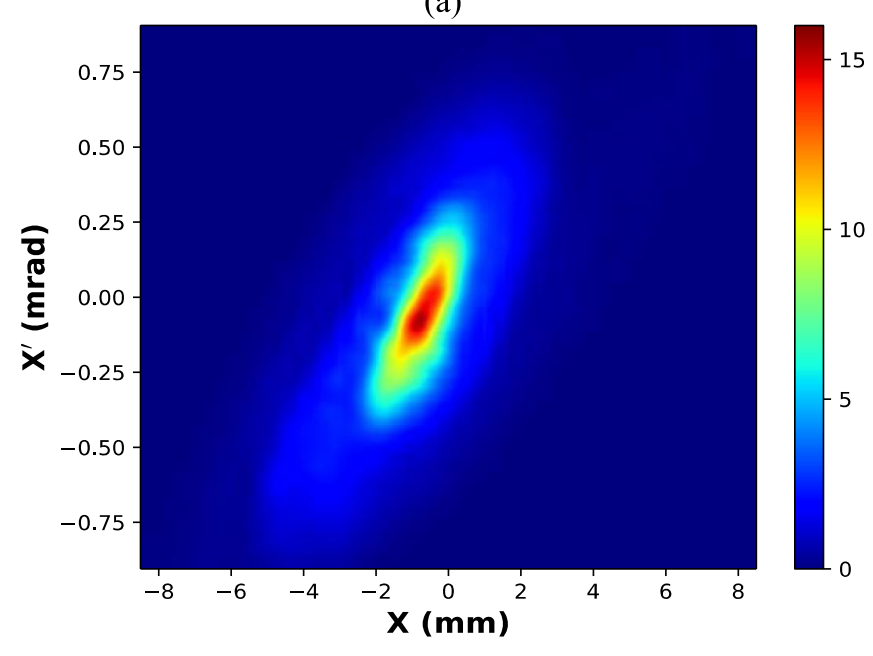

therefore can only be conducted on a $50 \mu \mathrm{s} / 1 \mathrm{~Hz}$ beam. The laser comb used in this measurement consists of 27 comb teeth with a spacing of $33 \mu \mathrm{s}$ (equivalent to the length of 35 minipulses). In the initial measurement, we skipped the ramp-up portion (100 minipulses) in the macropulse of the $\mathrm{H}^{-}$beam by aligning the first laser comb tooth with the 101st minipulse of the $\mathrm{H}^{-}$beam. Hence, the laser comb teeth interact with the $\mathrm{H}^{-}$beam at the minipulse numbers $101+35 * N(N=0,1, \ldots, 26)$. Each laser comb tooth contains nine micropulses which are in phase with the bunches of the $\mathrm{H}^{-}$beam. The measured 27 phase space slices in both transverse directions are displayed in Fig. 10. It is clear that emittances after the ramp-up are quite uniform across the macropulse of the $\mathrm{H}^{-}$beam. We calculated the integrated beam intensity and Twiss parameters from the measured phase space data and part of the results are plotted in Fig. 11. The rms emittance values are calculated from the phase space data by using the intensity mode method in Ref. [1] with a cutoff threshold set at 5\% of the peak intensity. The intensity variation among the minipulses is quite small after the initial ramp-up. Meanwhile, the emittance values vary about $20 \%$ over the macropulse and the variation patterns are similar between horizontal and vertical directions. The measured emittance values are consistent with the calculated values from wire scanners as well as with the design emittance.

The next measurement is of the phase space variation over the ramp-up region of the $\mathrm{H}^{-}$beam macropulse. The ramp-up process involves a continuous expansion of the minipulse duration at the beginning of the $\mathrm{H}^{-}$beam macropulse. For this measurement, the laser comb teeth spacing was changed to five minipulses and the first comb tooth is aligned with the first minipulse in the macropulse

FIG. 9. Measured phase space distributions in horizontal (a) and vertical (b) planes from one laser comb tooth. Viewing from upstream, horizontal scan is conducted from left $(-)$ to right $(+)$ and vertical scan is conducted from bottom $(-)$ to top $(+)$ in all emittance graphs. 
(a)

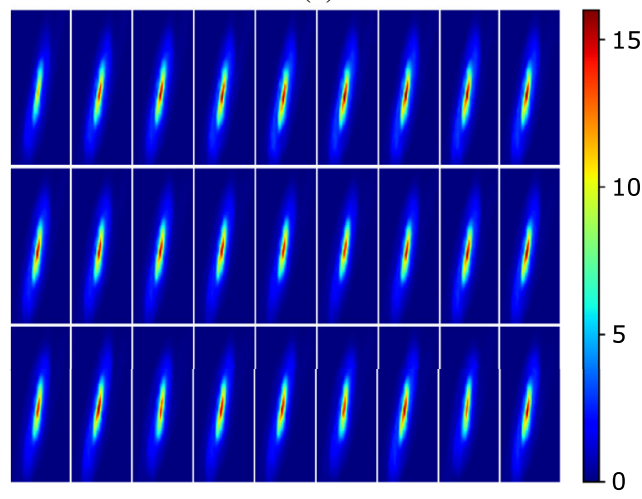

(b)

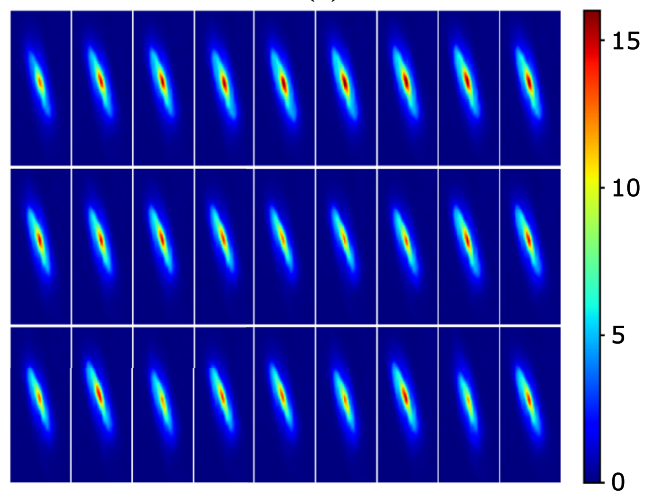

FIG. 10. Measured 27 slices of the phase space distributions of the 1.4-MW neutron production $\mathrm{H}^{-}$beam using a laser comb with the comb teeth spacing of $33 \mu$ s (35 minipulses). (a) Horizontal phase space. (b) Vertical phase space.

(a)

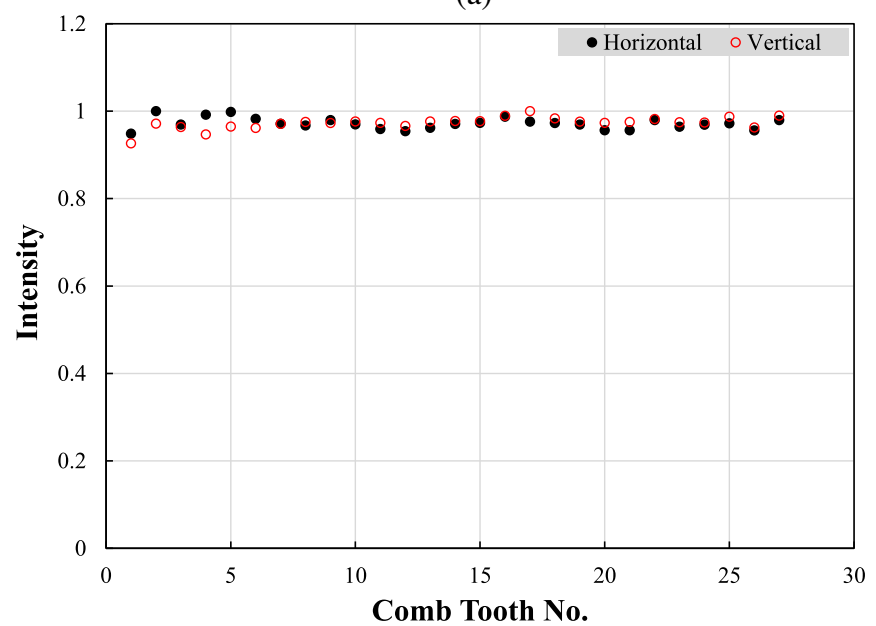

(b)

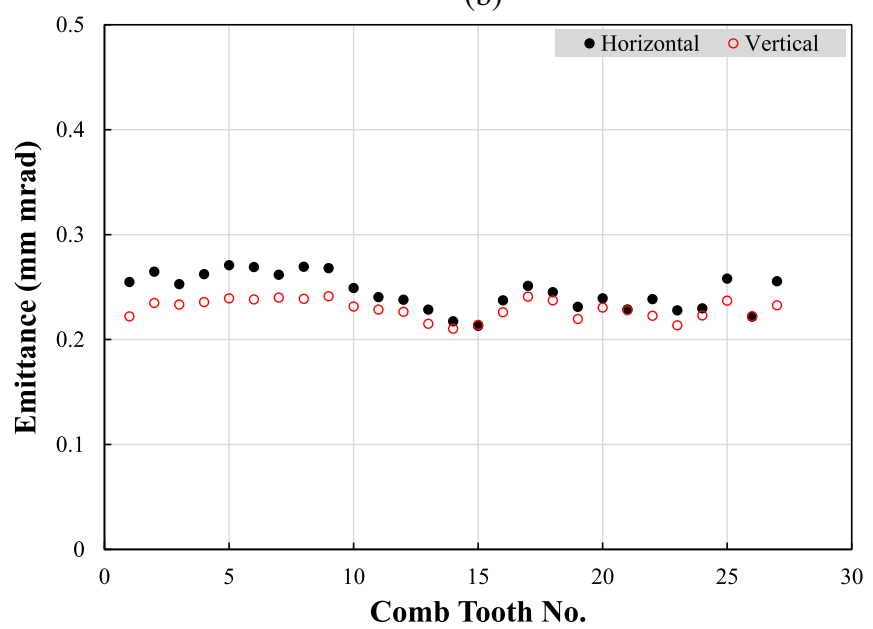

FIG. 11. Calculated beam parameters from the phase space data in Fig. 10. (a) Normalized intensities. (b) Emittance values.

(a)

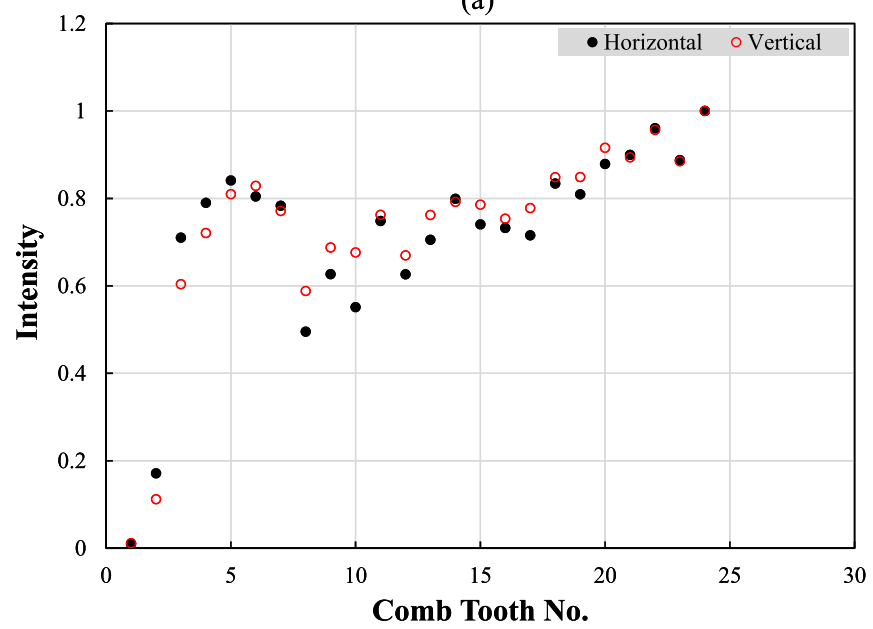

(b)

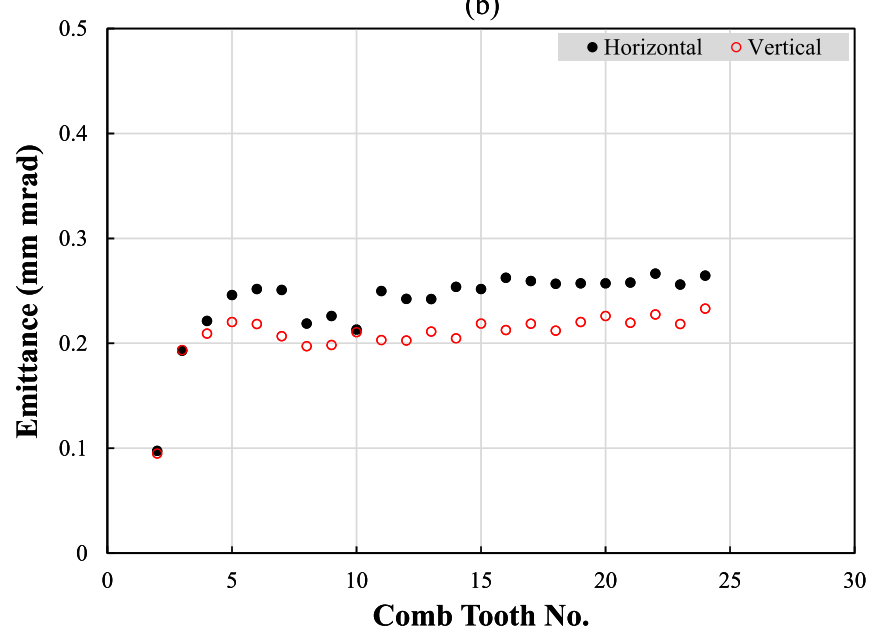

FIG. 12. Calculated beam parameters from the phase space distributions measured within the ramp-up of the 1.4-MW $\mathrm{H}^{-}$beam macropulse. Time difference between comb teeth is $\sim 4.7 \mu \mathrm{s}$ (5 minipulses). (a) Normalized intensities. (b) Emittance values. 
(a)

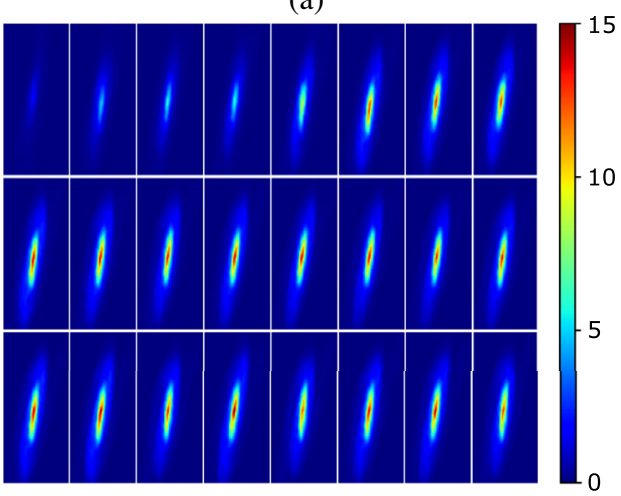

(b)

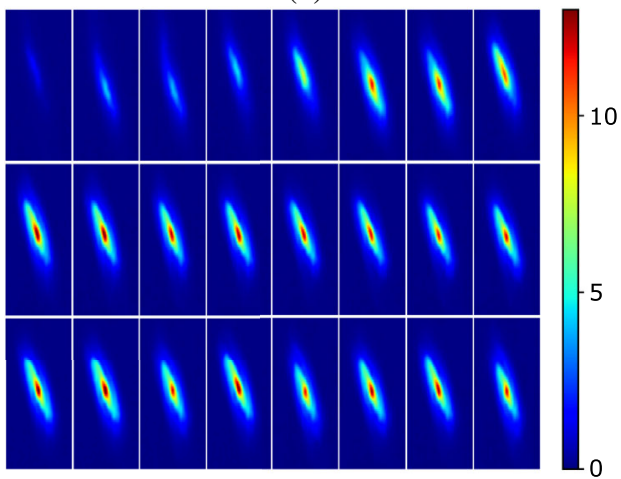

FIG. 13. Measured 24 slices of the phase space distributions along the rising edge of the 1.4-MW $\mathrm{H}^{-}$beam minipulse. The time difference between emittance slices is $\sim 3.3$ ns. (a) Horizontal phase space. (b) Vertical phase space.

of the $\mathrm{H}^{-}$beam. From the measured phase space data (not shown), we calculated beam parameters as plotted in Fig. 12. As expected, a monotonic increase of the beam intensity is observed during the beam ramp-up. Especially at the beginning of the beam ramp-up, the beam intensity shows a fast surge, which agrees well with the measurements from the beam current monitors. Meanwhile, the emittance values show a quick growth during the first 20 minipulses and stabilize after that. The center beam position (not shown) is quite steady in both directions. The minimum comb tooth spacing is limited by the available gain in the laser amplifiers. This limitation will be removed in the future with a planned installation of an additional laser amplifier, which will enable simultaneous emittance scans at each minipulse of the $\mathrm{H}^{-}$beam.

\section{Emittance scan over the rising edge of the $\mathrm{H}^{-}$beam minipulse}

In the above measurements, the laser comb tooth repetition rate was set as a subharmonic of the $\mathrm{H}^{-}$beam

(a)

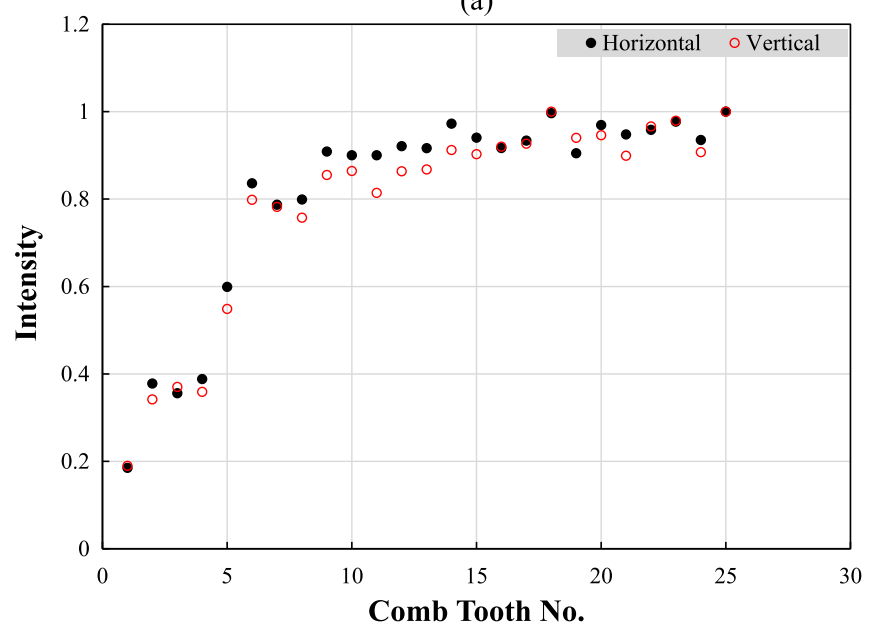

minipulse. Therefore, all comb teeth interact with the $\mathrm{H}^{-}$ beam at exactly the same position within the minipulse. In the following experiment, we demonstrate that the timeresolved emittance measurement can be performed over a single minipulse of the $\mathrm{H}^{-}$beam by slightly shifting the comb tooth frequency from the $\mathrm{H}^{-}$beam minipulse frequency. An example of 24 phase space slices measured by the laser comb is shown in Fig. 13. Here, the laser comb tooth repetition rate is set to $30.273 \mathrm{kHz}$ which is $3 \mathrm{~Hz}$ lower than the 35 th subharmonic of the $\mathrm{H}^{-}$beam minipulse frequency in Table I. As a result, each laser comb tooth interacts with the $\mathrm{H}^{-}$beam minipulse at $3.3 \mathrm{~ns}$ later than its predecessor. It is noted that the laser micropulses are still in phase with the ion beam bunches in this case. Therefore, the actual laser-ion interaction time remains the same for all comb teeth. The beam parameters calculated from the phase space data are shown in Fig. 14. We observe a gradual increase of the image brightness which indicates the transients of the ion beam intensity on the front edge of minipulses. The beam center position is steady in the horizontal direction but shows a sub-mm variation in the

(b)

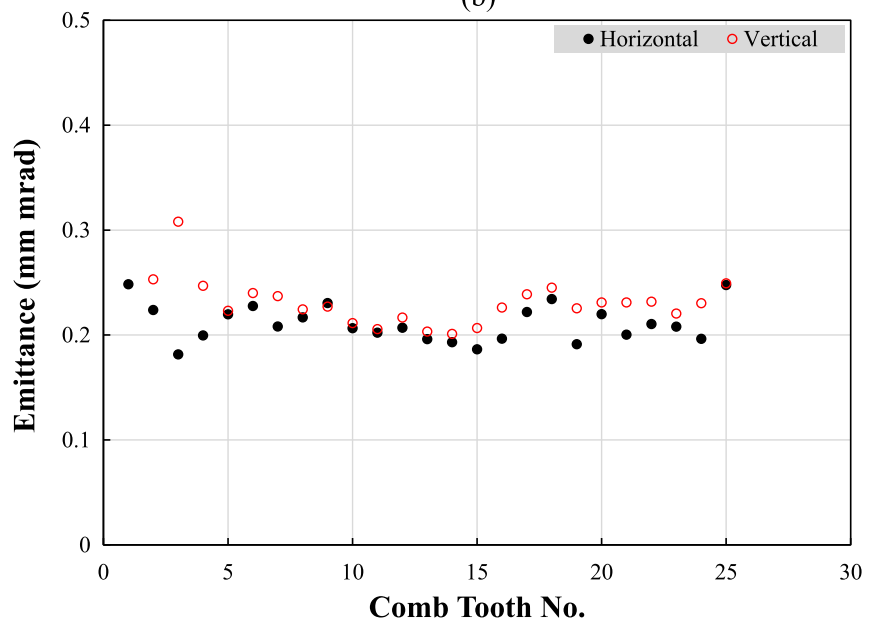

FIG. 14. Calculated beam parameters from the emittance data in Fig. 13. (a) Normalized intensities. (b) Emittance values. 
(a)

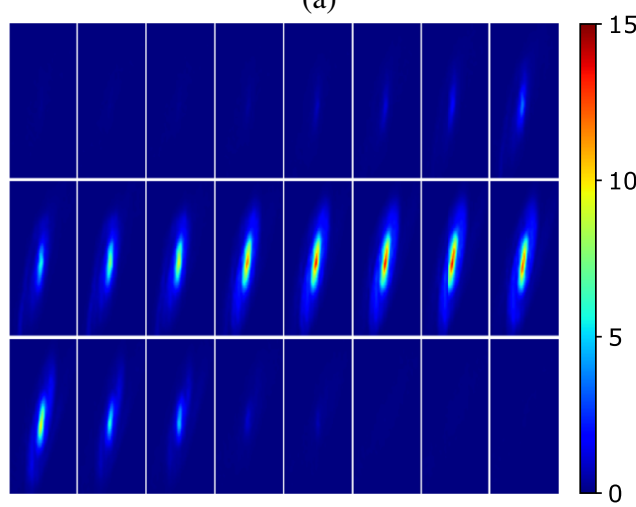

(b)

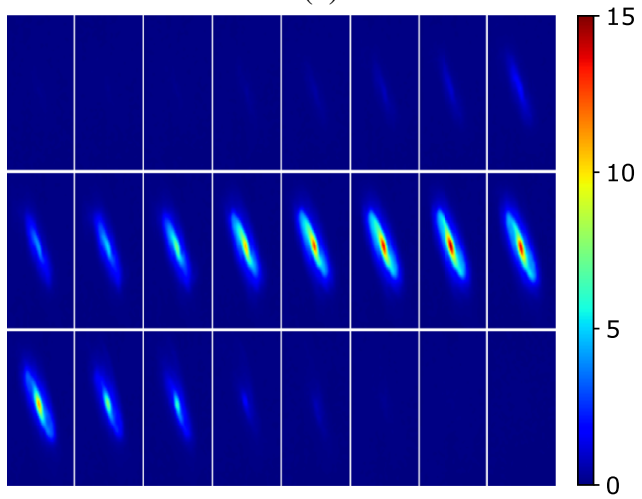

FIG. 15. Measured 24 slices of the phase space distributions over a single bunch of the 1.4-MW neutron production $\mathrm{H}^{-}$beam using laser comb. The time delay between emittance slices is 20 ps. (a) Horizontal phase space. (b) Vertical phase space.

vertical direction at the beginning of pulse. The calculated emittance values show some fluctuation at the beginning of the minipulse but quickly stabilize as displayed in Fig. 14(b).

\section{E. Emittance scan over a bunch of the $\mathrm{H}^{-}$beam}

Finally, we demonstrate the time-resolved phase space measurement over a single bunch of the $\mathrm{H}^{-}$beam by using a laser comb. The frequency shift approach employed in the previous experiment no longer applies here since the bunches of the $\mathrm{H}^{-}$beam are not synchronized to the macropulses or minipulses of the $\mathrm{H}^{-}$beam. Instead, we scan the phase of the micropulses inside a laser comb tooth by modulating the phase shifter using the signal from SG1 in Fig. 4 that controls the delay between the seed laser and the $\mathrm{rf}$ reference of the $\mathrm{H}^{-}$beam. As a result of the phase scan, the phase difference between the micropulse of the laser and the bunch of the $\mathrm{H}^{-}$beam is linearly tuned so that each laser comb tooth will interact with the $\mathrm{H}^{-}$beam at a different phase relationship in the micropulse (bunch) level. It is noted that the phase scan is only applied within a macropulse duration, i.e., $1 \mathrm{~ms} / 30 \mathrm{~Hz}$, and the phase scanning rate is very small $\left(\sim 10^{-6}\right)$. The phase difference between adjacent laser micropulses within a comb tooth is at the femtosecond level, which is negligible compared to the pulse width ( $>10 \mathrm{ps}$ ) of the $\mathrm{H}^{-}$beam bunch. Therefore, it can be well assumed that all micropulses inside one laser comb tooth are interacting with the $\mathrm{H}^{-}$beam bunches at the same phase. During the phase scan, the laser comb teeth are synchronized to the macropulses or minipulses of the $\mathrm{H}^{-}$beam.

Figure 15 shows the measured emittance slices over a single bunch of the $\mathrm{H}^{-}$beam. We observe a continuous variation of the image brightness from the rising edge to the falling edge of the $\mathrm{H}^{-}$beam bunch. As the scanning range is wider than the pulse width of the $\mathrm{H}^{-}$bunch, no valid measurement is anticipated near both edges of the scan. The calculated beam parameters are summarized in Fig. 16. (a)

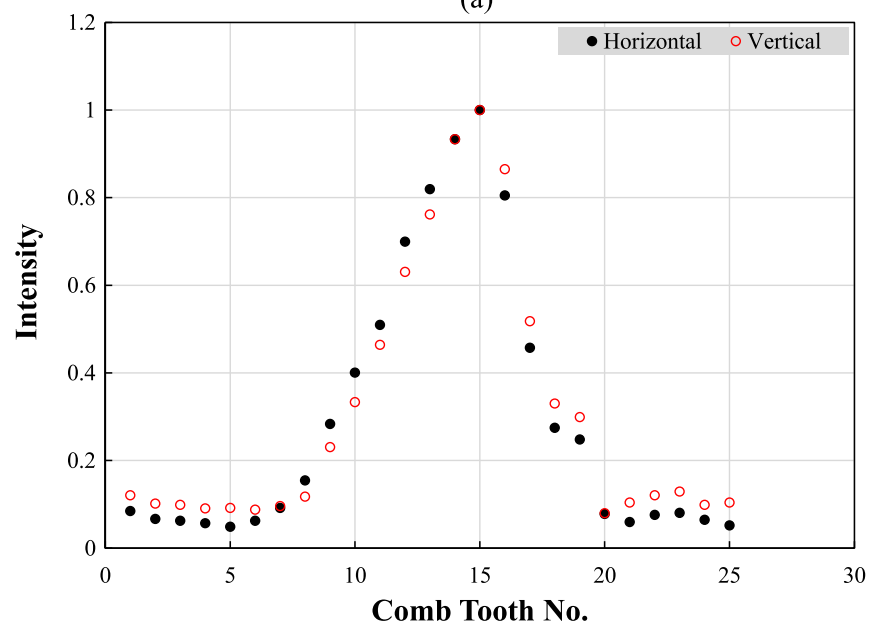

(b)

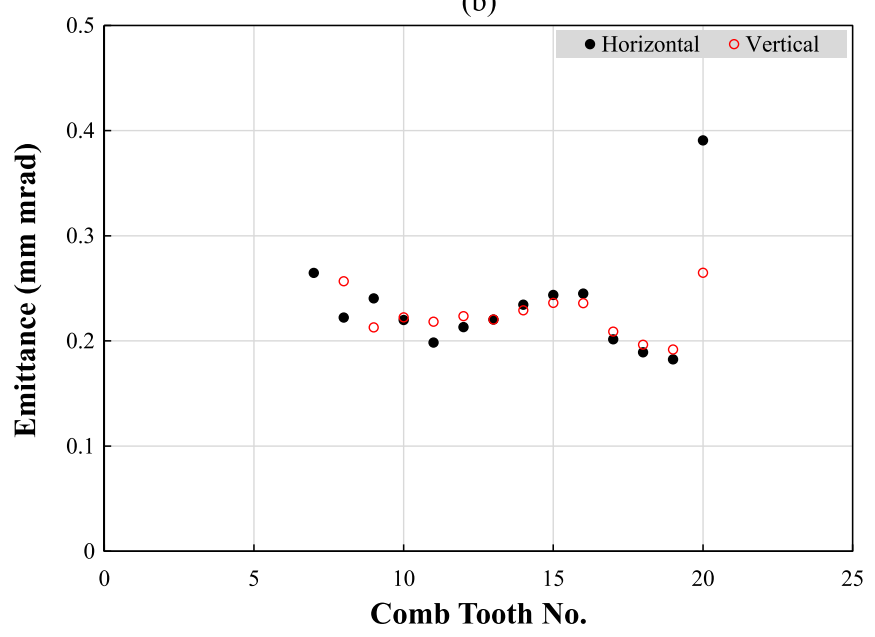

FIG. 16. Calculated beam parameters from the phase space data in Fig. 15. (a) Normalized intensities. (b) Emittance values. In (b), only the data from valid measurements (with sufficient intensities) are plotted. 
The intensity variations shown in Fig. 16(a) represent a longitudinal shape of the $\mathrm{H}^{-}$beam bunch within the resolution defined by the laser micropulse width and laser beam size. We estimated the rms bunch width to be about 50 ps which is consistent with the measurement results from the bunch shape monitor at the HEBT. The horizontal and vertical emittance values show a similar variation pattern. The variation is relatively small $(\sim 10 \%-15 \%)$ within the center of the bunch but increases substantially at the edges of the bunch. This is the first time that the change of the phase space distributions over a single bunch has been experimentally measured in a high energy, high power particle accelerator.

\section{CONCLUSIONS AND OUTLOOK}

We have described a novel technique of time-resolved emittance measurement of the $\mathrm{H}^{-}$beam by using a laser comb-laser pulses with a controllable multilayer pulse structure. The measurement is nonintrusive and can be performed on high-power operational $\mathrm{H}^{-}$beams. A laser system based on a mode-locked fiber laser and diodepumped solid-state laser amplifiers has been developed to generate laser combs with variable parameters. The proposed technique has been applied to the emittance measurement on the $1-\mathrm{GeV}, 1.4-\mathrm{MW}$ neutron production $\mathrm{H}^{-}$beam in the SNS high energy beam transport line. We have experimentally demonstrated that more than 20 slices of emittances over a macropulse, a single minipulse, or even a single bunch of the $\mathrm{H}^{-}$beam can be simultaneously obtained. Our experiment strongly supports the use of laser-based nonintrusive beam diagnostics to provide unique information about the high-energy, high-power particle beam.

While not explicitly described, the laser comb has also been successfully applied to the simultaneous measurement of time-resolved profiles of the operational $\mathrm{H}^{-}$beam at different time scales. Also as shown in Fig. 16, the laser comb can be potentially used to measure the bunch profile on a real-time basis. In principle, the phase delay can be set at a much finer step $(\sim 1 \mathrm{ps})$ using the current phase shifter. The limiting factor in the present laser setup is the laser pulse width ( $25 \mathrm{ps} \mathrm{rms)}$. Such a constraint can be resolved by replacing the seed laser in the present system with a picosecond pulsed light source. Finally, by applying the laser comb at different locations along the accelerator it may be feasible to also measure longitudinal phase space parameters of a high-power operational $\mathrm{H}^{-}$beam in real time.

In this paper, we mainly focused on the measurement technique itself. A detailed investigation of the emittance evolution on different time scales is a subject of a dedicated study. One immediate result is that the core rms emittance is fairly constant along the 1-ms pulse. This justifies the usage of the measurements of a narrow portion of the pulse with interceptive diagnostics in some accelerator sections where the laser wire is not available. Another important observation is the absence of significant rms emittance increase on the edges of the minipulses. Such an increase was observed [12] in the time-resolved emittance measurements in the SNS injector. The discrepancy could be possibly due to the insufficient dynamic range of the current laser comb measurements. Or, more interestingly, it could be explained by the large emittance growth in the main portion of the minipulse during the transport from the injector to the HEBT, which eliminated the emittance spikes on the minipulse edges. Along with the existing intercepting diagnostics in the injector, the time-resolved emittance measurement at the end of the linac provides a unique opportunity to experimentally address the above questions.

DOE will provide public access to these results of federally sponsored research in accordance with the DOE Public Access Plan [17].

\section{ACKNOWLEDGMENTS}

Support from S. Cousineau is acknowledged. We thank A. Webster and S. Murray III for their technical help and J. Holmes for his help with the manuscript. This research used resources at the Spallation Neutron Source, a DOE Office of Science User Facility operated by the Oak Ridge National Laboratory. This manuscript has been authored by UT-Battelle, LLC, under Contract No. DE-AC0500OR22725 with the U.S. Department of Energy (DOE). The U.S. government retains and the publisher, by accepting the article for publication, acknowledges that the U.S. government retains a nonexclusive, paid-up, irrevocable, worldwide license to publish or reproduce the published form of this manuscript, or allow others to do so, for U.S. government purposes.

[1] P. Strehl, Beam Instrumentation and Diagnostics (Springer-Verlag, Berlin, Heidelberg, 2006), Chap. 6.

[2] W. B. Cottingame, G. P. Boicourt, J. H. Cortez, W. W. Higgins, O. R. Sander, and D. P. Sandoval, Non-interceptive techniques for the measurement of longitudinal parameters for intense $\mathrm{H}^{-}$beams, IEEE Trans. Nucl. Sci. 32, 1871 (1985).

[3] R. C. Connolly, K. F. Johnson, D. P. Sandoval, and V. Yuan, A transverse phase-space measurement technique for high-brightness $\mathrm{H}^{-}$beams, Nucl. Instrum. Methods Phys. Res., Sect. A 312, 415 (1992).

[4] V. W. Yuan, R. C. Connolly, R. C. Garcia, K. F. Johnson, K. Saadatmand, O. R. Sander, D. P. Sandoval, and M. A. Shinas, Measurement of longitudinal phase space in an accelerated $\mathrm{H}^{-}$beam using a laser-induced neutralization method, Nucl. Instrum. Methods Phys. Res., Sect. A 329, 381 (1993).

[5] C. Gabor, H. Klein, O. Meusel, U. Ratzinger, and J. Pozimski, Experimental results of a non-destructive emittance measurement device for $\mathrm{H}^{-}$beams, in Proceedings of 
the 21st Particle Accelerator Conference, Knoxville, TN, 2005 (IEEE, Piscataway, NJ, 2005), p. 782, https://doi.org/ 10.1109/PAC.2005.1590562.

[6] Y. Liu, A. Aleksandrov, S. Assadi, W. Blokland, C. Deibele, W. Grice, C. Long, T. Pelaia, and A. Webster, Laser wire beam profile monitor in the spallation neutron source (SNS) superconducting linac, Nucl. Instrum. Methods Phys. Res., Sect. A 612, 241 (2010).

[7] Y. Liu, A. Aleksandrov, C. Long, A. Menshov, J. Pogge, A. Webster, and A. Zhukov, Nonintrusive emittance measurement of $1 \mathrm{GeV} \mathrm{H}^{-}$beam, Nucl. Instrum. Methods Phys. Res., Sect. A 675, 97 (2012).

[8] T. Hofmann, K. O. Kruchinin, A. Bosco, S. M. Gibson, F. Roncarolo, G. Boorman, U. Raich, E. Bravin, J. K. Pozimski, A. Letchford, and C. Gabor, Demonstration of a laserwire emittance scanner for hydrogen ion beams at CERN, Phys. Rev. ST Accel. Beams 18, 122801 (2015).

[9] H. Sakai et al., Measurement of a small vertical emittance with a laser wire beam profile monitor, Phys. Rev. ST Accel. Beams 5, 122801 (2002).

[10] I. Agapov, G. A. Blair, and M. Woodley, Beam emittance measurement with laser wire scanners in the International Linear Collider beam delivery system, Phys. Rev. ST Accel. Beams 10, 112801 (2007).

[11] A. P. Zhukov and A. V. Aleksandrov, High resolution emittance measurements at SNS front end, in Proceedings of LINAC2012, Tel Aviv, Israel, 2012 (JACoW, CERN, Geneva, Switzerland, 2012), p. 870.

[12] A. V. Aleksandrov, W. Blokland, Y. Liu, C. D. Long, and A. P. Zhukov, Diagnostics tools for beam halo investigation in SNS linac, in Proceedings of LINAC2012, Tel Aviv, Israel, 2012 (JACoW, CERN, Geneva, Switzerland, 2012), p. 873.

[13] E. Prat, M. Aiba, S. Bettoni, B. Beutner, S. Reiche, and T. Schietinger, Emittance measurements and minimization at the SwissFEL Injector Test Facility, Phys. Rev. ST Accel. Beams 17, 104401 (2014).

[14] B. Cathey, S. Cousineau, A. Aleksandrov, and A. Zhuko, First Six Dimensional Phase Space Measurement of an Accelerator Beam, Phys. Rev. Lett. 121, 064804 (2018).

[15] D. E. Johnson, T. R. Johnson, C. Bhat, S. Chaurize, K. Duel, P. Karns, W. Pellico, B. A. Schupbach, K. Seiya, and D. Slimmer, MEBT laser notcher (chopper) for booster loss reduction, in Proceedings of HB2018, Daejeon, Korea, 2018 (JACoW, CERN, Geneva, Switzerland, 2018), p. 416.

[16] Y. Liu, A. Rakhman, C. Long, Y. Liu, and T. Williams, Laser-assisted high-energy proton pulse extraction for feasibility study of co-located muon source at the SNS, Nucl. Instrum. Methods Phys. Res., Sect. A 962, 163706 (2020).

[17] http://energy.gov/downloads/doe-public-access-plan 